\title{
Horizons/Théâtre
}

Revue d'études théâtrales

10-11 | 2017

Genre et arts vivants

\section{Le metteur en scène vu comme un capitaine de navire}

Une métaphore discursive du modernisme théâtral et sa déclinaison à travers le $\mathrm{Xx}^{\mathrm{e}}$ siècle selon les perspectives de la génération et du genre

\section{Roberta Gandolfi}

\section{(2) OpenEdition}

12 Journals

Édition électronique

URL : http://journals.openedition.org/ht/612

DOI : $10.4000 /$ ht.612

ISSN : $2678-5420$

Éditeur

Presses universitaires de Bordeaux

\section{Édition imprimée}

Date de publication : 1 juillet 2017

Pagination : 308-318

ISSN : 2261-4591

\section{Référence électronique}

Roberta Gandolfi, « Le metteur en scène vu comme un capitaine de navire », Horizons/Théâtre [En ligne], 10-11 | 2017, mis en ligne le 01 juillet 2018, consulté le 20 juillet 2019. URL : http:// journals.openedition.org/ht/612 ; DOI : 10.4000/ht.612

\section{(c) (†) $\ominus$}

La revue Horizons/Théâtre est mise à disposition selon les termes de la Licence Creative Commons Attribution - Pas d'Utilisation Commerciale - Pas de Modification 4.0 International. 


\section{ROBERTA GANDOLFI}

Roberta Gandolfi est docteure en Études théâtrales et chercheuse à l'Université de Parme, où elle enseigne le théâtre contemporain et l'histoire de la mise en scène. Ses contributions, qui portent sur la mise en scène théâtrale et sur le champ du « théâtre et des études de genre » incluent les volumes, Un'Istruttoria lunga più di trent'anni. Olocausto, memoria, performance al Teatro Due di Parma, Milano, Mimesis, 2016, Un teatro attraversato dal mondo. II Théâtre du Soleil, oggi, Pisa, Titivillus, 2012 (co-autrice avec Silvia Bottiroli) et La Prima regista. Edith Craig, fra rivoluzione della scena e cultura delle donne, Roma, Bulzoni, 2003, ainsi que de nombreux articles parus dans des revues italiennes et internationales (Biblioteca Teatrale, II Castello di Elsinore, Teatro e Storia, European Journal of Women Studies, etc.)

\section{Mail : robertapierangela.gandolfi@unipr.it, rogandolfi@libero.it}

Résumé: Cette contribution concerne le discours sur la mise en scène, entre autoreprésentations des artistes et études critiques : comme s'est-il développé à partir du modernisme théâtral ? Quelles images et métaphores sont devenues canoniques et ont-elles été l'objet de négociations de la part des artistes et du discours historiographique et critique? Est-il possible de faire une analyse selon la perspective du genre, afin d'observer comment les metteurs et les metteuses en scène se sont positionné.e.s par rapport à ce discours ? J'analyse la célèbre métaphore du metteuren-scène comme capitaine d'un navire, qui nourrit la canonisation du metteur en scène en tant que leader du travail théâtral, à travers plusieurs exemples: Gordon Craig une empreinte fortement hiérarchique (il

Abstract: The essay concerns the categories and the images used in contemporary theatre to talk about directing. What canonical metaphors and images are used concerning the role of theatre directors? Is it possible to historicize such speech? How did it develop, from modernism onwards? And what happens if we assume the point of view of gender, and try to understand how male and female directors position themselves in relation to canonical metaphors? The metaphor taken in consideration is the one that parallels the director of a play to the captain of a ship: a powerful image, which contributed, from modernism onwards, to claim the hegemony of the director on the theatrical realm. The essay goes into its first formulation, which is due to Gordon Craig, and discusses the hierarchical connotation of parlait de "discipline » et " obéissance ») ; puis, la puissance normative de ce discours, qui parfois a amené les études théâtrales à une certaine cécité, voir une incapacité à reconnaitre d'autres modalités de création dans le théâtre contemporain; enfin, les ruses des metteurs en scène, hommes et femmes, de Peter Brook à Ariane Mnouchkine, pour reconfigurer la métaphore du capitaine de navire, au-delà de sa connotation hiérarchique et genrée (un homme aux commandes), et pour l'adapter à différentes manières de faire et logiques créatives.

Mots-clés: Mise-en-scène, Capitaine de navire, Gordon Craig, Peter Brook, Ariane Mnouchkine

his speech, with its claim of « discipline » and " obedience »; it argues the power of such speech and its regulatory effect on theatre studies; it addresses some side effects, like the reluctance of theatre studies to acknowledge different types of leadership into the realm of the " directors theatres ». Finally, it identifies different ways through which directors, male and female - from Peter Brook to Ariane Mnouchkine - reconfigured the same metaphor to speak about their work, going beyond the masculine and hierarchical connotation (one man at command), and enriching it with new meaning.

Keywords: Director, Captain of the ship, Gordon Craig, Peter Brook, Ariane Mnouchkine 


\section{Le metteur en scène vu comme un}

\section{capitaine de navire}

\section{Une métaphore discursive du modernisme théâtral et sa déclinaison à travers le}

\section{XXe siècle selon les perspectives de la génération et du genre}

Cet article pose la question de l'autorialité de la mise en scène à partir de l'analyse discursive de la figure du « capitaine de navire », une métaphore usitée depuis le début du $\mathrm{Xx}^{\mathrm{e}}$ siècle en Europe et dont je rappellerai ici les fondements historiques et son utilisation par plusieurs metteurs et metteuses en scène au cours du temps.

En effet, au cours de mes recherches, j'ai pu remarquer qu'il existe des images puissantes apparues sous la forme de métaphores récurrentes qui se sont sédimentées dans le champ discursif de la mise en scène et qui fonctionnent comme des aimants identitaires. Un de ces aimants est celui qui concerne le metteur en scène vu comme un capitaine de navire.

Ainsi, je m'attache à analyser le discours sur la mise en scène, entre autoreprésentations des artistes, études critiques et historiographie : comment s'est-il développé à partir du modernisme théâtral ${ }^{1}$ ? Comment s'est-il décliné à travers le théâtre, du $\mathrm{Xx}^{\mathrm{e}}$ siècle jusqu'à nos jours ?

Il s'agit d'une constellation discursive assez complexe, car elle a évolué dans le temps et concerne plusieurs aspects : éthique, esthétique et politique. Elle touche au rôle et à la fonction de la mise en scène, soit par rapport aux modes de production et de création inhérents aux métiers du théâtre, soit par rapport au théâtre en tant que medium de communication et de symbolisation. Quelles sont, dans le domaine de la mise en scène théâtrale, les formations discursives devenues canoniques et objet de constantes renégociations de la part des artistes et du discours historiographique et critique ? Est-il possible de faire une analyse selon la perspective du genre, d'observer comment les metteurs et les metteuses en scène se sont positionné.e.s par rapport à ces discours, comment ils et elles l'ont assumé, modifié et réinscrit dans les autoreprésentations de leur travail et de leur métier?

J'ai d'ores et déjà développé ces questions au sein de mon travail d'historienne de la mise en scène théâtrale. J'ai souvent privilégié l'étude des femmes metteuses en scène européennes ; en particulier dans deux ouvrages dédiés à Edith Craig ${ }^{2}$ et au Théâtre du Soleil d'Ariane Mnouchkine ${ }^{3}$, comblant ainsi 
un vide qui persistait dans les études italiennes sur la mise en scène, qui privilégient l'analyse et le récit de l'autorialité masculine dans le théâtre contemporain. Dans d'autres ouvrages de nature historiographique ${ }^{4}$, j'ai cherché à mettre en évidence la façon dont de telles orientations patriarcales ont produit une narration canonique de l'histoire de la mise en scène, une narration qu'il est temps aujourd'hui de déconstruire. Considérant les stratégies de cette branche de l'édition théâtrale au sujet du phénomène de la mise en scène, $j$ 'ai souligné comment, dans tous les pays européens, sont apparues des séries de publications monographiques dédiées aux grands metteurs en scène, en tant qu'auteurs/héros du théâtre contemporain (comme la série « Directors in Perspective » de la Cambridge University Press), qui ont construit progressivement un « panthéon de la mise en scène » rigide et fermée ${ }^{5}$. Ce panthéon historiographique peut être considéré comme une narration « mythique » de l'autorialité de la mise en scène, dans la mesure où elle part du présupposé idéologique et non problématisé qui célèbre le Metteur en scène en tant qu'Auteur, l'Artiste du théâtre contemporain ${ }^{6}$. Il me semble que peu de travaux articulent de façon critique cette question de fond qui touche aux modalités de travail dans la mise en scène, c'est-à-dire celle de l'autorialité. $\mathrm{Au}$ sein de cet art intrinsèquement collectif, les conditions d'exercice et de légitimation du leadership de la mise en scène apparaissent nombreuses et complexes ; si on les interroge de manière critique, sans considérer le metteur en scène comme une image « mythique »d'homme aux manettes, elles peuvent nous apprendre beaucoup sur les nouvelles pratiques de la création théâtrale du $\mathrm{Xx}^{\mathrm{e}}$ siècle.

\section{Gordon Craig : le metteur en scène, capitaine de navire}

La métaphore du metteur en scène vu comme un capitaine de navire fut proposée par Gordon Craig dans son célèbre traité publié en 1905 : On the Art of the Theatre - first dialogue (paru en France en 1916 sous le titre De l'art $d u$ théâtre). L'auteur introduit dans le débat esthétique du théâtre moderne la nécessité de la mise en scène. Craig la considérait comme le nouvel art du théâtre :

Le Régisseur : «J'attends une Renaissance. 》

L'Amateur de théâtre: «Et qu'est-ce que qui l'amènera?»

Le Régisseur : « L'avènement d'un homme réunissant en sa personne toutes les qualités qui font un maître du théâtre et la rénovation du théâtre en tant qu'instrument ${ }^{7}$ » 
Dans ce texte, Craig compare la figure et le rôle du metteur en scène à un chef d'orchestre ou à un capitaine de navire, en insistant tout particulièrement sur l'organisation hiérarchique et la discipline devant exister entre tous les corps de métier afin d'en garantir la bonne navigation.

À la fin d'un long dialogue entre un «stage-manager » (régisseur) et un « playgoer » (spectateur), le premier affirme : « Pensez un instant aux relations hiérarchiques des hommes à bord d'un bateau et vous saisirez mieux comment j'envisage celles qui unissent entre eux les gens du théâtre ${ }^{8}$. »

Puis : « Mais ce que je tiens à vous montrer, c'est que tant qu'on ne comprendra pas que la discipline au théâtre consiste en l'obéissance volontaire, absolue au directeur de la scène - équivalent du capitaine - on ne pourra rien entreprendre de grand $^{9}$. »

La métaphore du metteur en scène vu comme un capitaine de navire est peut-être la première et la plus célèbre image qui lie l'autorialité de la scène à une idée d'autorité et de leadership formulée selon un modèle très rigide et hiérarchique : le metteur en scène, en tant qu'auteur, est l'homme seul qui dirige, l'homme seul aux commandes.

Avec cette métaphore, Gordon Craig a voulu revendiquer la suprématie de la nouvelle figure de l'auteur : en l'occurrence le metteur en scène, au détriment des figures hégémoniques du théâtre du XIX ${ }^{\mathrm{e}}$ siècle, c'est-à-dire les acteurs et les dramaturges ${ }^{10}$.

Rappelons que si l'image du metteur en scène/capitaine de navire a connu un grand succès durant les premières décennies $\mathrm{du} \mathrm{xx}^{\mathrm{e}}$ siècle, c'est parce qu'à l'époque, les metteurs en scène menaient un véritable combat pour affirmer leur hégémonie au sein des institutions théâtrales européennes. Mais, en même temps, cette image puissante contribua à éclipser d'autres façons moins hiérarchiques de mettre en scène, d'autres lieux moins institutionnels où le nouvel art de la scène se forgeait, et d'autres individus qui ne se reconnaissaient pas forcément dans cette figure masculine.

Il faut considérer, à ce propos, ce que Charlotte Canning a écrit sur la façon dont la revue moderniste Theatre Arts construit le discours sur la mise en scène pendant les années ving $\mathrm{t}^{11}:$ la chercheuse américaine a mis en évidence comment la revue avait privilégié des portraits individuels dédiés aux personnalités de la mise en scène - un homme seul aux commandes, selon notre métaphore - plutôt que des articles dédiés aux contextes opérationnels de l'art theatre, des little theatres et des community theatres, où beaucoup de femmes étaient promotrices et metteuses en scène. 
Les femmes metteuses en scène à l'époque des avant-gardes étaient peu nombreuses. Les femmes directrices furent une exception, où trouvaient-elles, alors, leur légitimation sociale et culturelle pour exercer un rôle de leadership artistique ? Leur possibilité et légitimité d'action, comme je l'ai écrit dans mon livre sur Edith Craig, se lie au contexte historique. Les premières décennies du siècle dernier ont vu l'affirmation de nouveaux sujets politiques : les mouvements émancipationnistes, qui ont conquis l'accès des femmes au vote politique, et les mouvements des ouvriers, protagonistes des luttes politiques et syndicales. Edith Craig, sœur de Gordon, inventrice d'un théâtre d'art et militante, tirait la légitimité de son leadership du mouvement suffragiste ; 20 ans plus tard, pendant les années trente, Joan Littlewood commençait son travail théâtral avec le Workers' Theatre Movement, en connexion avec le Parti Communiste, en même temps que, aux États-Unis, Hallie Flanagan était à la direction du Federal Theatre Project (1935-1939), un projet très ambitieux de théâtre populaire.

Leurs théâtres furent engagés dans des luttes et des discours collectifs, et leur travail de mise en scène visait à la valorisation de ces nouveaux sujets sociaux ; leurs formidables inventions théâtrales n'avaient rien en commun avec le modèle de fonctionnement hiérarchique proposé par Gordon Craig. Toutefois, leur contribution esthétique et politique à l'art de la mise en scène a été effacée par les historiens du théâtre et par le discours moderniste, lorsqu'on a considéré l'émergence de cette nouvelle instance artistique, la mise en scène, comme l'émergence d'individualités créatrices singulières qui ont développé un style individuel et une signature esthétique d'auteur (comme la métaphore du metteur en scène capitaine de navire nous le signale).

En d'autres termes, la métaphore du metteur en scène comme capitaine de navire a permis à l'art naissant de la mise en scène d'être plus visible et reconnaissable alors qu'elle s'exprimait en syntonie avec le canon moderniste de l'auteur (très fort dans le modernisme littéraire). Quand, au contraire, l'art de la mise en scène se liait aux pratiques performatives anti-autoriales de nombreuses avant-gardes esthétiques et politiques, elle a été moins reconnaissable ${ }^{12}$.

\section{Peter Brook et Ariane Mnouchkine : un capitaine de navire genré}

On remarque que ce sont le plus souvent les hommes que les femmes qui utilisent, directement ou indirectement, l'expression « capitaine de navire » pour parler de la question du leadership. Et si la métaphore devient souvent un «lieu commun », elle peut aussi devenir le point de départ d'une reformulation des modèles de leadership différents. 
En 1980, Peter Brook reformule la définition de Gordon Craig tout en y faisant écho :

On doit distinguer deux sens dans l'expression « mettre en scène ». La moitié de la tâche du metteur en scène consiste, bien sûr, à diriger, à prendre en charge, à décider, à dire «oui » et à dire «non», à avoir le dernier mot. L'autre moitié consiste à maintenir la bonne direction. Là, le metteur en scène - le director devient un guide, il tient le gouvernail, il doit avoir étudié les cartes et savoir s'il se dirige vers le nord ou vers le sud ${ }^{13}$.

Dans la définition de Peter Brook, l'image du capitaine de navire est indirecte mais bien présente («the director is at the helm », il tient le gouvernail, il est à la barre... ). Le « sens de la direction » (que Brook oppose dans ce même essai à la « directorial conception », à la conception directoriale) fait évoluer la métaphore du metteur en scène/capitaine de navire vers l'image d'un mouvement, d'une recherche : en plus de commander, le rôle d'un capitaine de navire est d'indiquer la direction du voyage. Cette connotation (mettre en scène, c'est être en voyage) permet à Brook d'exprimer sa propre vision et façon d'exercer son influence, tout comme les metteurs en scène des ensembles théâtraux de la seconde moitié du $\mathrm{Xx}^{\mathrm{e}}$ siècle (du Berliner Ensemble dirigé par Bertolt Brecht, à la Schaubhüne dirigée par Peter Stein) : celle d'un guide d'un ensemble d'artistes, d'un processus collectif - et pas individuel - de création ; au début du voyage c'est le metteur en scène qui entrevoit la route (comme une intuition informe, « a formless hunch $\gg$ ), mais le voyage est à faire ensemble ${ }^{14}$.

Que se passe-t-il alors, dans le cas, rare à ma connaissance, où la métaphore est utilisée par une metteuse en scène $\mathrm{e}^{15}$ ? Un siècle après Gordon Craig, Ariane Mnouchkine la reformule à son tour.

Il y a quelques années, en 2008, au cours d'un cycle de rencontres avec des élèves du Conservatoire National Supérieur d’Art Dramatique, elle a déclaré : «Vous avez charge d'âmes. J'ai charge d'âmes comme un capitaine de bateau. Quand on ouvre la porte au public on a charge d'âmes à tout point de vue... et du point de vue de leur sécurité, et du point de vue de leur espoir dans l'humanité ${ }^{16} \gg$.

Mnouchkine s'identifie ici, en tant que metteuse en scène et leader du Théâtre du Soleil, à la figure influente et masculine du capitaine de bateau, mais elle l'enrichit avec détermination d'une dimension éthique. Bien que Mnouchkine soit athée, l'expression « vous avez charge d'âmes » évoque un pasteur protestant face à ses fidèles et à son rôle de guide spirituel : les âmes 
évoquées dans cette métaphore, ce sont les spectateurs qu'il faut accueillir et nourrir à la source du théâtre.

Donc, Mnouchkine ne refuse pas la métaphore du metteur en scène/capitaine de navire, mais elle rend ses implications plus complexes, selon une logique genrée. Le thème de l'autorialité théâtrale entendue comme discipline et commandement (la métaphore de Gordon Craig) se transforme avec Mnouchkine en thème de l'autorialité théâtrale entendue comme responsabilité d'accueillir. Avec cette métaphore, Mnouchkine déplace l'idée de la direction de toutes celles et tous ceux qui sont sur le pont (les actrices et les acteurs, les technicien.ne.s) vers l'idée de la direction des passagers, les voyageurs du théâtre, son public.

En 2008, quand Mnouchkine se sert de cette métaphore, on peut supposer que son imaginaire ne se réfère pas directement à la définition de Gordon Craig, mais plutôt au travail de la troupe du Soleil qui à ce moment-là est en pleine création du spectacle Les Naufragés du Fol Espoir ${ }^{17}$. Ce spectacle, l'histoire d'une utopie, montre une équipe de cinéma, en vogue au seuil de la Grande Guerre, qui filme l'aventure d'un bateau faisant naufrage en Terre de feu. Mnouchkine exprime ainsi la même vision que celle de Peter Brook: diriger une troupe de théâtre, diriger une équipe de cinéma ou bien encore diriger un navire, cela signifie être en voyage, à la recherche d'un monde nouveau.

De plus, pour Mnouchkine, faire monter les voyageurs sur le bateau est la métaphore et l'image verbale qu'elle choisit pour raconter ce qu'elle accomplit chaque jour de spectacle au Soleil, en tant que metteuse en scène, depuis longtemps, depuis toujours : ouvrir la porte au public et donner la main à chacun des spectateurs. Ainsi, ce n'est pas un hasard si Les Naufragés du Fol Espoir nous offre cette belle scène de l'embarquement des passagers pour un long voyage vers un autre continent...

On s'aperçoit que toutes les configurations discursives qui concernent le travail de création au Théâtre du Soleil convergent vers une éthique de l'accueil. « Accueillir » est un mot-clé au Soleil, et ce, à tous les niveaux de la création théâtrale : Mnouchkine, en tant que leader, accueille les propositions des acteurs, l'écrivaine Hélène Cixous raconte qu'elle aussi accueille les personnages en faisant le vide en elle, et les acteurs sont invités à accueillir leurs personnages, mais aussi leurs compagnons de travail ou le moment présent ${ }^{18}$.

Cependant, cette éthique de l'accueil et de la réception n'est pas exclusivement du côté du féminin : disons qu'elle dynamise le masculin et le féminin, le corps et l'esprit, l'imagination et la relation aux autres, elle entremêle 
l'anima et l'animus, le concave, le poreux et l'élan vers l'effort, vers l'élévation, vers la transformation alchimique de la matière narrative, imaginative, pulsionnelle et affective dont est fait le théâtre.

Pour conclure, je voudrais suggérer que le discours sur la mise en scène n'est pas seulement fait de canonisations ou de contestations. On peut doncle déconstruire sans toutefois le banaliser, afin d'en montrer toutes les nuances et de l'interroger selon une perspective de genre. Avec le temps et au travers de la subjectivité sexuée des artistes - c'est-à-dire des hommes et des femmes qui reprennent ce discours et le recréent -, la figure du capitaine de navire se stratifie et devient plus complexe : pour Gordon Craig, le capitaine est le leader, l'homme aux commandes ; pour Peter Brook, il est celui qui indique la direction du voyage et, pour Ariane Mnouchkine, il.elle est celui-celle qui accueille, qui reçoit, tout en ayant une responsabilité artistique envers les spectateurs, les voyageurs, les citoyens du théâtre.

À propos de public et de spectateurs, je voudrais revenir juste un instant à Gordon Craig. Dans son traité, en effet, le spectateur est déjà présent, puisque Craig en fait le partenaire privilégié de son dialogue. Mais le spectateur de Gordon Craig était un homme ignorant, qu'il fallait éduquer, à qui il fallait expliquer l'art du théâtre. Alors qu'au Soleil, le spectateur est une foule, à la fois singulière et plurielle, composée d'hommes et de femmes. Et pour Ariane Mnouchkine, tout l'enjeu artistique est de se placer à la hauteur de l'humanité des spectateurs, de leurs espoirs et de leurs utopies.

\section{Notes}

1. En général, on applique l'étiquette de modernisme aux expérimentations littéraires anglophones et à l'art visuel, ainsi qu'à l'architecture des avant-gardes artistiques continentales. Par exemple, l'anthologie de Vassiliki Lolocotroni, Jane Goldam et Olga Taxidou, Modernism: An Anthology of Sources and Documents, Chicago, Chicago Press, 1998, met en parallèle des documents de l'avant-garde historique et des parties du modernisme littéraire. La scène et l'écriture théâtrales furent impliquées de différentes façons tant avec le modernisme littéraire qu'avec les avant-gardes - dada, futurisme, surréalisme, expressionisme. Lorsque je parle de modernisme théâtral, je ne me réfère donc pas à un phénomène esthétique unitaire, mais à la possibilité de concevoir différentes innovations des arts performatifs qui ont caractérisé les deux dernières décennies du $\mathrm{XX}^{\mathrm{e}}$ siècle et les trois premières décennies du $\mathrm{XXI}^{\mathrm{e}}$, sous l'angle des expérimentations modernistes. Dans Modernism and Performance. Jarry to Brecht, New York, Palgrave and Macmillian, 2007, Olga Taxidou montre les nombreuses façons par lesquelles dans cette période historique sur les scènes anglaises et continentales a été mise en forme et conceptualisée la notion de "modernist performance », et rappelle l'importance de 
"the radical reworking of the "professions" of the theatre ", parmi lesquels " the rise of the director ", ibid., p. XVI, pour le modernisme théâtral. Dans ce sens, j'adhère à l'approche d'Olga Taxidou et considère en outre que l'avènement de la profession du metteur en scène peut être tenue pour un trait caractérisant le modernisme théâtral.

2. Roberta Gandolfi, La Prima regista. Edith Craig, fra rivoluzione della scena e cultura delle donne, Rome, Bulzoni, 2003.

3. Silvia Bottiroli et Roberta Gandolfi, Un teatro attraversato dal mondo. Il Théâtre du Soleil, oggi, Pise, Titivillus, 2012.

4. Roberta Gandolfi, " Gli studi sulla regia teatrale ", Annali di Lettere $\mathrm{n}^{\circ} 1$, Università degli Studi di Ferrara, 2006, p. 237-253 (en ligne : http://eprints.unife.it/annali/ lettere) ; Roberta Gandolfi, «Quando la ricerca teatrale incontra gli studi delle donne e di genere ", Biblioteca Teatrale n ${ }^{\circ} 105-106,2014$, p. 141-152.

5. Sur le concept de " panthéon de la mise en scène », voir Roberta Gandolfi, « La regia estesa: una prassi refrattaria al pantheon de la mise en scène ", dans La prima regista, op. cit., p. 179-204, et Roberta Gandolfi, "Gli studi sulla regia teatrale », op. cit. Je renvoie également à l'article de Denis Kennedy, "The Director, the Spectator and the Eiffel Tower ", Theatre Research International $n^{\circ} 30,2005$, p. 36-48, qui prend en considération l'actualité théâtrale : avec l'inclusion des étoiles émergentes de la mise en scène, aujourd'hui le panthéon de la mise en scène fonctionne souvent comme une formule de référence pour construire les programmes européens des festivales du théâtre.

6. Déjà en1980, Jean-Jacques Roubine soulignait comment l'adresse monographique qui attribue uniquement au « génie » du metteur en scène « la paternité de tout ce qui est produit au théâtre » suscite généralement une approche idéaliste (Théâtre et mise en scène, 1880-1980, Paris, PUF, 1980, p. 11). Aujourd'hui, certaines études remarquent qu'il faut décrire le processus de création des "théâtres de régie » (directors theatres), comme un travail d'équipe (team work) et pas comme le travail d'un individu, seul, aux commandes (Maria Delgado, Dan Rebellato, Contemporary European Theatre Directors, Londres, Routledge, 2010, p. 21).

7. Edward Gordon Craig, De l'art du théâtre, trad. par Geneviève Séligmann-Lui, introduction de Jacques Rouché, Paris, éd. Nouvelle Revue Française, 1916, p. 168-169. Le texte original, en anglais (paru en 1905) : "Stage manager: "But I look for a renaissance"। Playgoer: "How will that come?" / Stage manager: "Through the advent of a man who shall contain in him all the qualities which go to make up a master of the theatre, and through the reform of the theatre as an instrument" ", J. Michael Walton (ed.), Craig on Theatre, Londres, Methuen, 1991, p. 69.

8. Edward Gordon Craig, De l'art du théâtre, op. cit., p. 162. Le texte original, en anglais : "Stage manger: "Consider for a moment the relationship of the men on a ship, and you will undestand what I consider to be the relationship of men in a theatre" ", J. Michael Walton (ed.), Craig on Theatre, op. cit., p. 66.

9. Edward Gordon Craig, De l'art du théatre, op. cit., p. 164. Le texte original, en anglais: "Stage manager: "What I wish to show you is that until discipline is understood in a theatre to be willing and reliant obedience to the manager or captain no supreme achievement can be accomplished" ", J. Michael Walton (ed.), Craig on Theatre, op. cit., p. 67.

10. Dans la célèbre formulation de Gordon Craig, l'art du théâtre n'est pas l'art de l'acteur ni l'art du dramaturge, mais bel et bien l'art du metteur en scène. Ce dernier se concentre sur le dynamisme et le mouvement des corps, des objets et de la narration. Il met en 
espace mais il n'est pas sur scène, et il crée à travers l'action, les paroles, la ligne, les couleurs et le rythme. Il est l'auteur de la scène, il est le nouvel artiste. Les acteurs, les techniciens, le texte, sont tous à son service.

11. Charlotte Canning, "Directing History: Women, Performance and Scholarship ", Theatre Research International $\mathrm{n}^{\circ}$ 1, vol. 30, 2005, p. 49-59.

12. On sait bien que le modernisme ne fut pas seulement un, il fut à la fois le temps d'une expérimentation formelle et d'une expérimentation politique, en connexion avec le débat littéraire et avec les bouleversements politiques et sociaux : voir, à ce propos, Olga Taxidou, Modernism and Performance, op. cit. Sur l'effacement des expérimentations modernistes des artistes femmes de théâtre (dramaturges, actrices et metteures en scène), voir Katherine Kelly, Modern Drama by Women, 1880s-1930s : An International Anthology, Londres, Routledge, 1996 ; Penny Farfan, Women, Modernism and Performance, Cambridge, Cambridge University Press, 2004, et mon livre La Prima regista. Edith Craig, fra rivoluzione della scena e cultura delle donne, op. cit.

13. Peter Brook, Points de suspension : quarante-quatre ans d'exploration théâtrale, trad. JeanClaude Carrière et Sophie Reboud, Paris, Seuil, 2004, p. 18. La version originale, en anglais : "I think one must split the word "direct" down to the middle. Half of directing is, of course, being a director, which means taking charge, making decision, saying "yes" and saying "no", having the final say. The other half of directing is maintaining the right direction. Here, the director becomes guide, he's at the helm, he has to have studied the maps and he has to know whether he's heading north or south ", Peter Brook, "The formless hunch ", 1980, dans son livre The Shifting Point, Londres, Methuen Drama, 1989, p. 5.

14. Avec l'expression " ensembles théâtraux » (dans le sillage de l'historien du théâtre Claudio Meldolesi, auteur avec Laura Olivi de l'ouvrage Brecht regista. Memorie del Berliner Ensemble, Bologna, Il Mulino, 1989), je me réfère à certaines grandes formations européennes de la seconde moitié $\mathrm{du} \mathrm{xx}^{\mathrm{e}}$ siècle, où la question du leadership s'articule et se décline selon des modes coopératifs de création : parmi eux le Berliner Ensemble, la Schaubhüne, le CIRT, le Taganka, le Théâtre du Soleil, etc. Sur les modalités de création de type coopératif, et sur les pratiques de mise en scène caractéristiques de ces « théâtres d'ensembles ", j'ai dédié l'essai, «Processi creativi: il teatro d'ensemble e le scritture collettive » dans le volume Silvia Bottiroli e Roberta Gandolfi (dir.), Un teatro attraversato dal mondo. Il Théâtre du Soleil oggi, op. cit., p. 160-182.

15. Outre Ariane Mnouchkine, sur laquelle je me concentrerai dans les pages qui suivent, je signale au moins une autre directrice qui fait référence à la métaphore du capitaine de navire (je dois cette découverte à un article récemment paru de Laura Mariani, « Registe di teatro in Italia. Ce n'est qu'en début », Culture teatrali n² 25, 2016, p. 108-123). Dans une interview réalisée avec Andrea Porcheddu et Patrizia Bologna, la sicilienne Emma Dante explique que son travail créatif avec sa compagnie a peu à faire avec les modalités classiques de direction scénique qui partent d'une lecture du texte à froid, guidée par un « capitaine » : dans son cas « l'ovulation a lieu sur scène, il n'y a pas de conception extérieure », Andrea Porcehddu (dir.), Emma Dante. Palermo dentro, Arezzo, Zona, 2010, p. 42.

16. Voir le volume édité par Béatrice Picon-Vallin, Ariane Mnouchkine, Arles, Actes-Sud Papiers, 2009, p. 70.

17. Voir mon chapitre, «Les Naufragés du Fol Espoir », dans Silvia Bottiroli et Roberta Gandolfi, Un teatro attraversato dal mondo. Il Théâtre du Soleil, oggi, op. cit., p. 109-134. 
18. Pour un approfondissement et une contextualisation des discours féministes, voir Roberta Gandolfi, «Fra Ariane Mnouchkine e Hélène Cixous. Poetiche e pratiche di genere nei processi creativi al Théâtre du Soleil ", dans Saveria Chemotti (dir.), Affettività elettive. Relazioni e costellazioni disordinate, Padoue, Il Poligrafo, 2014, p. 159-168. Pour la question du féminisme et de la place d'Ariane Mnouchkine dans ce champ, voir Raphaëlle Doyon, Roberta Gandolfi, "Mnouchkine, Ariane ", dans Christine Bard, Sylvie Chaperon (dir.), Dictionnaire des féministes. France XVIII ${ }^{-}$ XXI siècle, Paris, PUF, 2017. 